\title{
Utilização da Realidade Virtual como ferramenta para inclusão de indivíduos com Transtorno do Espectro Autista no mercado de trabalho: uma revisão sistemática
}

\author{
Giulia T. Monteiro e Diana F. Adamatti \\ Centro de Ciências Computacionais - Universidade Federal do Rio Grande (FURG) \\ Caixa Postal 474 - 96.203-900 - Rio Grande - RS - Brazil \\ \{giuliatondin, dianaadamatti\}@ furg.br

\begin{abstract}
This paper presents a systematic review on the insertion of young adults with Autistic Spectrum Disorder (ASD) in the labor market, making use of Virtual Reality as a support tool. Performing a systematic review is a methodologically correct way of working, trying to bring the greatest amount of work in a scientific way. The goal of this paper is to analyze the work that is done in the area and what are the advantages and disadvantages of using this technology to help this specific population. In this way, some comparison metrics between the works were defined. We can conclude that this area is not explored and it can have significant results to improve the quality of life and independence of individuals with ASD.
\end{abstract}

Resumo. Este artigo apresenta uma revisão sistemática sobre a inserção de jovens adultos com Transtorno do Espectro Autista (TEA) no mercado de trabalho, fazendo uso de Realidade Virtual como ferramenta de apoio. Realizar uma revisão sistemática é uma forma de trabalho metodologicamente correta, tentando trazer a maior quantidade de trabalhos de uma maneira científica. $O$ artigo tem por objetivo analisar os trabalhos que vem sendo realizados na área e quais as vantagens e desvantagens da utilização desta tecnologia para auxiliar essa população específica. Para tanto, algumas métricas de comparação entre os trabalhos encontrados foram definidas. Pode-se concluir que essa é uma área ainda pouco explorada e que pode ter resultados significativos para melhoria da qualidade de vida e independência de indivíduos com TEA.

\section{Introdução}

O Transtorno do Espectro Autista (TEA) se refere a uma série de condições caracterizadas por algum grau de comprometimento no que foi definido como Tríade das Incapacidades [Wing and Gould 1979]. Esta tríade é representada pelas funções de comunicação, socialização e imaginação, fazendo com que o indivíduo desempenhe um comportamento repetitivo e estereotipado. Foi observado que aproximadamente uma a cada 68 crianças foram diagnosticadas com o transtorno nos Estados Unidos [Baio 2014], estimandose a existência de 2 milhões de pessoas somente no Brasil [Giannini 2018]. Porém, apesar dos números crescentes de diagnósticos, estudos apontam que a inclusão nas configurações da sociedade, como escola e mercado de trabalho, é dificilmente alcançada [Hendricks 2010, Shattuck et al. 2012].

Shattuck el al. (2012) demonstrou que apenas 55\% dos jovens adultos com TEA conseguem emprego após o término do ensino médio. Indivíduos diagnosticados com 
autismo de alto funcionamento e Síndrome de Asperger possuem o maior risco de desemprego, chegando a ter três vezes mais chances de não se inserir no ambiente laboral ou outras atividades diurnas na comunidade do que indivíduos com TEA que possuem alguma deficiência intelectual [Taylor and Mailick 2011]. Este contexto coloca muitos desses indivíduos em dificuldades financeiras, ainda que apresentem formação e capacidade para trabalhar, tornando-os dependentes de familiares e instituições [Bontempo 2009].

Nos últimos anos, a tecnologia tornou-se uma grande aliada para o aprimoramento de habilidades em indivíduos com TEA, tanto crianças quanto adultos. $\mathrm{O}$ grande interesse dessa população por dispositivos eletrônicos, em partes, deve-se ao fato de uma melhor resposta aos estímulos advindos dessas tecnologias que dos oriundos das pessoas [Miller and Bugnariu 2016]. Nos últimos anos, estudos sugeriram que a Realidade Virtual (RV) é uma ferramenta particularmente útil para essa população [Parsons and Mitchell 2002, Kandalaft et al. 2012].

Revisões sistemáticas fornecem acesso à evidências pré-filtradas, sintetizando os resultados de múltiplos estudos originais utilizando estratégias para definir os dados metodologicamente mais fortes e relevantes [Schlosser 2006]. Ao responder questões específicas de uma determinada área, as revisões reduzem de modo considerável o tempo e conhecimento necessários para reunir e avaliar todas as pesquisas.

Deste modo, o objetivo deste artigo é realizar uma revisão sistemática de forma a localizar e organizar estudos que tratem sobre a inserção de jovens adultos com Transtorno do Espectro Autista no mercado de trabalho, utilizando a RV como ferramenta de apoio, a fim de definir o que está sendo trabalhado nesta área de pesquisa.

\section{Realidade Virtual e TEA}

A Realidade Virtual surgiu como uma nova ferramenta de apoio eficaz em diferentes tópicos da área da saúde, como na saúde mental. Nela, os pacientes podem utilizar um ambiente seguro e controlado durante o tratamento e acompanhamento de transtornos como fobias, Transtorno Obsessivo-Compulsivo, Transtorno de Estresse Pós-Traumático e, claro, Transtorno do Espectro Autista [Aresti and Zapirain 2014]. Ou seja, indivíduos com TEA podem praticar interações e comportamentos dentro de um ambiente realista, ensaiando e repetindo cenários em diferentes contextos, possibilitando a generalização das habilidades aprendidas no ambiente virtual para o cotidiano [Tzanavari et al. 2015, Didehbani et al. 2016].

A RV pode ser caracterizada pela coexistência de três aspectos: imersão, interação e envolvimento [Morie 1994]. Sendo imersão o sentimento de fazer parte do ambiente; interação a capacidade do computador detectar as entradas do usuário e possuir capacidade reativa; e envolvimento o grau de motivação para a participação de uma pessoa em determinada atividade.

\subsection{Realidade Virtual Imersiva e Não Imersiva}

Pode-se diferenciar RV imersiva de não imersiva da seguinte maneira: a primeira é obtida através do uso de capacete de visualização (Head-mounted Display) ou de uma Caverna (sistemas imersivos que projetam a simulação nas paredes, teto e piso de uma sala), enquanto a segunda apenas visualiza o ambiente através de monitores/telas de televisão, porém mesmo assim cria uma sensação de presença e realidade [Leston 1996]. Outros 
VIII Congresso Brasileiro de Informática na Educação (CBIE 2019)

Anais do XXX Simpósio Brasileiro de Informática na Educação (SBIE 2019)

sentidos, além da visão, podem ser estimulados para proporcionar algum grau de imersão para a RV que utiliza monitores, como a audição [Robertson et al. 1993].

Nos estudos envolvendo RV e indivíduos com TEA, a segunda definição foi mais empregada ao estimular outros sentidos, porém a abordagem imersiva possui grande potencial e interesse crescente de pesquisa. Desse modo, busca-se analisar estudos que levam em consideração diferentes graus de imersão, porém possuem um objetivo em comum: aumentar a inclusão de jovens adultos com TEA no mercado de trabalho.

\section{Metodologia Utilizada para Revisão Sistemática}

\subsection{Critério de seleção}

Para definir estratégias na busca de evidências foi utilizado o modelo PICO [Roever 2018], que tem como objetivo identificar os quatro elementos fundamentais da questão da pesquisa, sendo eles: População/Paciente, Intervenção, Comparação e Outcomes (desfecho). Sendo assim, foi estabelecido o seguinte PICO para a questão da pesquisa:

- População: jovens adultos com TEA;

- Intervenção: treinamento para o mercado de trabalho utilizando Realidade Virtual;

- Comparação: condição do indivíduo antes do tratamento com Realidade Virtual;

- Outcomes: Principais resultados obtidos.

Os estudos devem apresentar iniciativas eficientes e eficazes de ambientes em Realidade Virtual como ferramenta de suporte para inclusão de jovens adultos com TEA no mercado de trabalho. Porém, devido à limitações de pesquisa, a maioria dos estudos tem curta duração, limitando assim os resultados obtidos.

\subsection{Estratégia de pesquisa}

Foram realizadas pesquisas de literatura nas seguintes base de dados: Science Direct ${ }^{1}$, Scopus $^{2}$, PubMed Central (PMC) ${ }^{3}$, SpringerLink ${ }^{4}$, IEEEXplore ${ }^{5}$ e SciELO ${ }^{6}$. Estas bases de dados foram escolhidas por serem as maiores nas áreas de computação e ciências médicas, que são as duas áreas envolvidas na pesquisa. A única base que possui trabalhos em português é a SciELO, mas não foram obtidos trabalhos em português. Desta forma, para pesquisa, os termos foram pesquisados para palavras-chave em inglês.

Apenas artigos dos últimos dez anos (a partir de 1 de janeiro de 2009 até 31 de janeiro de 2019) foram observados na revisão, visto que pesquisas na área da Realidade Virtual tiveram um enorme avanço nos anos mais recentes.

As palavras-chave escolhidas foram: (autism) AND (youth OR adult OR "young adult") AND ("Virtual Reality"OR "3D user interface"OR "head-mounted display") AND ("job interview"OR "employment"OR "vocational training").

\footnotetext{
${ }^{1}$ https://www.sciencedirect.com/

${ }^{2}$ https://www.scopus.com/

${ }^{3}$ https://www.ncbi.nlm.nih.gov/pme/

${ }^{4}$ https://link.springer.com/

${ }^{5} \mathrm{https}$ ://ieeexplore.ieee.org/Xplore/home.jsp

${ }^{6}$ www.scielo.br/
} 
VIII Congresso Brasileiro de Informática na Educação (CBIE 2019)

Anais do XXX Simpósio Brasileiro de Informática na Educação (SBIE 2019)

\subsection{Processo de seleção}

O processo pode ser dividido em quatro fases. Primeiramente, foram realizadas buscas nas bases de dados utilizando os termos, ambos definidos anteriormente, resultando na seleção de 240 estudos. De modo a refinar a pesquisa, foram aplicados critérios de inclusão e exclusão no conjunto inicial, a partir da leitura do título e resumo de cada estudo selecionado, tais como: (1) o estudo foi aplicado à jovens adultos diagnosticados clinicamente com TEA? e (2) a intervenção utilizava RV como ferramenta de suporte para a inclusão no mercado de trabalho? Desta etapa restaram apenas 18 estudos a serem analisados.

Visto que alguns estudos não deixaram claro seus objetivos e metodologias, foi possível continuar o refinamento da pesquisa ao executar novamente os critérios primeiramente na introdução e logo após no texto completo. Parte dos estudos selecionados inicialmente deixaram em aberto o real funcionamento da solução, utilizando o termo "realidade virtual" para denominar um ambiente virtual de interação, mesmo esse não criando nenhuma sensação de presença no mesmo, critério necessário para uma aplicação de RV.

Ao final, apenas 6 estudos foram selecionados, sendo separados em duas categorias, imersiva e não imersiva. As etapas do processo podem ser observadas na Tabela $1 \mathrm{e}$ as respectivas categorias dos estudos na Tabela 2.

Tabela 1. Processo de seleção dos estudos da revisão

\begin{tabular}{cc}
\hline Fase & Total de estudos selecionados \\
\hline Conjunto Inicial & 240 \\
Título e Resumo & 18 \\
Introdução & 10 \\
Texto Completo & 6 \\
\hline
\end{tabular}

Tabela 2. Resultado final de estudos para diferentes tipos de RV

\begin{tabular}{cc}
\hline Tipo de RV & Total de estudos selecionados \\
\hline Imersiva & 1 \\
Não imersiva & 5 \\
\hline
\end{tabular}

\section{Resultados}

Dos 6 estudos selecionados a partir dos critérios de inclusão e exclusão, 1 estudo utilizou RV Imersiva na sua definição de pesquisa, suas características estão descritas na Tabela 3.

Enquanto que 5 estudos utilizaram RV Não Imersiva para sua aplicação, suas características podem ser observadas na Tabela 4.

\subsection{Métricas de avaliação}

Esta seção apresenta algumas métricas de comparação entre os 6 trabalhos selecionados pela revisão sistemática, abordando os objetivos de cada trabalho, bem como o número de participantes ou tipo de tecnologia utilizada, e finalizando pelas melhorias ou limitações encontradas. 
VIII Congresso Brasileiro de Informática na Educação (CBIE 2019)

Anais do XXX Simpósio Brasileiro de Informática na Educação (SBIE 2019)

Tabela 3. Resumo do estudo utilizando RV imersiva

\begin{tabular}{|c|c|c|c|c|}
\hline Referência & Foco & Participantes & Avaliação & Resultados \\
\hline [Schmidt et al. 2019] & $\begin{array}{l}\text { Aumentar a independên- } \\
\text { cia ao utilizar o transporte } \\
\text { público para ampliar o } \\
\text { acesso ao emprego e } \\
\text { comunidade. }\end{array}$ & $\begin{array}{l}5 \text { homens. } \\
\text { Idade: } 22-34 \text { anos. }\end{array}$ & $\begin{array}{c}\text { Análise a partir de dados } \\
\text { de revisão por especialis- } \\
\text { ta e testes de uso utilizan- } \\
\text { do técnicas quantitativas e } \\
\text { qualitativas. Análise dos pon- } \\
\text { tos da Escala de Usabilida- } \\
\text { de (SUS). }\end{array}$ & $\begin{array}{c}\text { Resultados da revisão de especia- } \\
\text { listas sugeriram que o aplicativo } \\
\text { é viável, relevante e fácil de usar. } \\
\text { No entanto, foram realizadas } \\
\text { uma série de orientações, inclu- } \\
\text { indo o equilíbrio entre usabili- } \\
\text { dade e imersão, para otimizar } \\
\text { a aprendizagem e questões } \\
\text { sensoriais para os indivíduos } \\
\text { com TEA. }\end{array}$ \\
\hline
\end{tabular}

\subsubsection{Quanto aos objetivos e as questões de pesquisa}

Os resultados demonstraram uma clara correlação entre os objetivos de 5 dos 6 estudos, onde focaram em analisar os efeitos das intervenções no aprimoramento de habilidades para entrevistas de emprego, visto que essa é uma das maiores barreiras enfrentadas para a entrada no mercado de trabalho [Shattuck et al. 2012].

[Burke et al. 2017] foca em duas importantes questões sobre a real eficácia desse método de intervenção para o aprimoramento de habilidades. Primeiramente, se os participantes com alguma deficiência de desenvolvimento ou com TEA tiveram uma diferença significativa no grau de utilização de determinada habilidade e, também, se existiu uma diferença estatisticamente considerável entre o início e final da intervenção.

O principal objetivo do estudo de [Strickland et al. 2013] foi analisar não apenas as habilidades dos participantes perante ao entrevistador, mas também como o conteúdo das respostas era entregue. Além disso, tinha por hipótese que existiria relação entre o grau de sintomas do TEA e o total de melhora obtida.

Também considerando o diagnóstico de TEA e aspectos como a teoria da mente, [Kandalaft et al. 2012] examinaram a viabilidade da intervenção proposta e as mudanças de comportamento social. Propuseram como hipótese que as habilidades de percepção e conversação social, reconhecimento de emoções e teoria da mente mudariam, analisando o desempenho antes e após o treinamento.

[Schmidt et al. 2019] trouxeram uma nova questão de pesquisa, buscando aumentar a inserção de jovens adultos no mercado de trabalho, a partir do aumento da independência na utilização de transporte público, visto que o transporte também é uma das barreiras mais citadas para a inclusão desses indivíduos, tanto no ambiente laboral quanto em outros contextos da sociedade. Suas principais questões focaram-se principalmente na experiência do usuário, na viabilidade, relevância e usabilidade do ambiente para indivíduos com TEA.

\subsubsection{Quanto ao número de participantes}

[Schmidt et al. 2019] utilizaram a menor amostra, com apenas 5 indivíduos com TEA que estavam inscritos no programa envolvido no estudo para realizar o teste de uso do ambiente. Com idades entre 22 e 34 anos, todos os participantes eram homens e faziam parte da faixa leve e moderada da Escala de Responsividade Social [Constantino et al. 2003], 
VIII Congresso Brasileiro de Informática na Educação (CBIE 2019)

Anais do XXX Simpósio Brasileiro de Informática na Educação (SBIE 2019)

Tabela 4. Resumo dos estudos utilizando RV não imersiva

\begin{tabular}{|c|c|c|c|c|}
\hline Referência & Foco & Participantes & Avaliação & Resultados \\
\hline [Ward and Esposito 2018] & $\begin{array}{c}\text { Examinar a relação } \\
\text { entre a utilização da } \\
\mathrm{RV} \text {, autoconfiança e } \\
\text { habilidades para en- } \\
\text { trevista pré e pós } \\
\text { intervenção. }\end{array}$ & $\begin{array}{c}\text { Grupos: } \\
10 \text { homens, } \\
2 \text { mulheres. } \\
\text { Idade: } 18-22 \text { anos. }\end{array}$ & $\begin{array}{c}\text { Escala Geral de } \\
\text { Autoeficácia (GSEF), } \\
\text { avaliação de autocon- } \\
\text { fiança, monitoramen- } \\
\text { to de progresso atra- } \\
\text { vés do ambiente. }\end{array}$ & $\begin{array}{l}\text { Melhoria das habilidades } \\
\text { de entrevista e autocon- } \\
\text { fiança, aumento dos ní- } \\
\text { veis de conforto em } \\
\text { indivíduos com autismo } \\
\text { de autofuncionamento. }\end{array}$ \\
\hline [Burke et al. 2017] & $\begin{array}{l}\text { Fornecer prática para } \\
\text { aumentar habilidades } \\
\text { em uma variedade de } \\
\text { condições em entrevis- } \\
\text { tas de emprego. }\end{array}$ & $\begin{array}{c}\text { Grupos: } \\
25 \text { homens, } \\
7 \text { mulheres. } \\
\text { Idade: } 19-31 \text { anos. } \\
70 \% \text { possuía TEA. }\end{array}$ & $\begin{array}{l}\text { Cálculo da diferença mé- } \\
\text { dia de pontuação entre } \\
\text { a primeira e a últi- } \\
\text { ma sessão e entre as } \\
\text { sessões consecutivas. }\end{array}$ & $\begin{array}{l}\text { Util para obter conheci- } \\
\text { mento e ampliar a com- } \\
\text { preensão dos participantes } \\
\text { sobre etiquetas e ex- } \\
\text { pectativas de entrevistas. }\end{array}$ \\
\hline [Smith et al. 2017] & $\begin{array}{l}\text { Aprender sobre as habi- } \\
\text { lidades de entrevista a } \\
\text { partir de aulas didáticas } \\
\text { online e praticar essas } \\
\text { habilidades durante inte- } \\
\text { rações com uma agente } \\
\text { virtual de recursos } \\
\text { humanos. }\end{array}$ & $\begin{array}{c}\text { Grupos: } \\
56 \text { homens, } \\
23 \text { mulheres. } \\
\text { Idade: } 18-55 \text { anos }\end{array}$ & $\begin{array}{c}\text { Avaliação pós teste } \\
\text { em nove domínios de } \\
\text { desempenho, avaliação de } \\
\text { neurocognição e estimador } \\
\text { bayesiano. }\end{array}$ & $\begin{array}{l}\text { Identificou a relação entre } \\
\text { o número de entrevistas vir- } \\
\text { tuais completas e melhores } \\
\text { habilidades de entrevista } \\
\text { com mecanismos para ob- } \\
\text { ter ofertas de emprego. }\end{array}$ \\
\hline [Strickland et al. 2013] & $\begin{array}{l}\text { Avaliar a eficácia de um } \\
\text { pacote de treinamento } \\
\text { composto por um pro- } \\
\text { grama de habilidades de } \\
\text { entrevista online e prá- } \\
\text { tica em RV em indivíduos } \\
\text { com autismo de alto fun- } \\
\text { cionamento e Síndrome } \\
\text { de Asperger. }\end{array}$ & $\begin{array}{l}22 \text { homens. } \\
\text { Idade: } 16-19 \text { anos. }\end{array}$ & $\begin{array}{l}\text { Avaliação de habilidades } \\
\text { em entrevistas, composta } \\
\text { por duas categorias: con- } \\
\text { teúdo de resposta e en- } \\
\text { trega de resposta. Escala } \\
\text { de Responsividade Social. } \\
\text { Informação demográfica. }\end{array}$ & $\begin{array}{l}\text { Demonstrou ser um } \\
\text { método útil, porém } \\
\text { foi mais eficaz no } \\
\text { ensino de habilidades } \\
\text { de 'conteúdo' } \\
\text { do que de 'entrega'. }\end{array}$ \\
\hline [Kandalaft et al. 2012] & $\begin{array}{l}\text { Investigar a viabilidade } \\
\text { de um Treinamento de } \\
\text { Cognição Social de RV } \\
\text { focada no aprimoramento } \\
\text { de habilidades sociais, } \\
\text { cognição social e } \\
\text { funcionamento social. }\end{array}$ & $\begin{array}{l}8 \text { participantes. } \\
\text { Idade: } 18-26 \text { anos. }\end{array}$ & $\begin{array}{c}\text { ACS-SP - Pearson, } \\
\text { Teoria da Mente, } \\
\text { Habilidades de conversa- } \\
\text { ção e pesquisa de acom- } \\
\text { panhamento. }\end{array}$ & $\begin{array}{l}\text { Aumentos significativos } \\
\text { nas medidas da teoria da } \\
\text { mente e reconhecimento } \\
\text { de emoções, bem como } \\
\text { no social e funcionamen- } \\
\text { to ocupacional. }\end{array}$ \\
\hline
\end{tabular}

indicando deficiências no comportamento social clinicamente significativas. Outro estudo que utilizou um menor tamanho de amostra, foi o de [Kandalaft et al. 2012], porém foi composto apenas por indivíduos com Síndrome de Asperger ou com PDD-OS (Transtorno global de desenvolvimento sem outra especificação), que possui sintomas similares aos do autismo. A faixa etária do estudo variou entre 18 e 26 anos, além de que sete dos participantes já estavam empregados ou estudavam em tempo integral.

[Strickland et al. 2013] também limitou o diagnóstico para a seleção dos participantes, selecionando apenas aqueles que possuíam autismo de alto funcionamento ou Síndrome de Asperger, além de terem sido desconsiderados todos aqueles que possuíam algum problema motor, de audição ou de visão. Ao todo, 22 homens participaram, com idades entre 16 e 19 anos.

A pesquisa de [Ward and Esposito 2018] trabalhou com 16 estudantes de uma escola pública da Califórnia, porém 4 não completaram todos os aspectos do estudo, sendo a única com desistência de participação, que teve como justifica incopatibilidades de agenda. Levando em consideração os que completaram, 10 identificaram-se como homens e 2 como mulheres, e a idade dos participantes variou entre 18 e 22 anos.

O estudo com a maior amostra foi realizado por [Smith et al. 2017], reunindo 79 indivíduos que pertenciam a uma gama de diagnósticos, incluindo TEA, não deixando claro qual tinha maior predominância, porém excluindo aqueles que possuíam alguma 
VIII Congresso Brasileiro de Informática na Educação (CBIE 2019)

Anais do XXX Simpósio Brasileiro de Informática na Educação (SBIE 2019)

doença médica que comprometesse a cognição. Também possuiu a maior faixa etária dos participantes, variando entre 18 e 55 anos. Todos estavam em situação de desemprego ou de subemprego, sendo que $44 \%$ dos indivíduos estavam desempregados entre 24 a 59 meses.

Outro estudo que utilizou diferentes diagnósticos no estudo foi o de [Burke et al. 2017], no qual reuniu 32 participantes com idades entre 19 e 31 anos e com diferentes deficiências de desenvolvimento. Ao todo, $70 \%$ da amostra era formada por indivíduos com TEA, além de ser composta por 25 homens e apenas 7 mulheres.

Em todos os estudos houve a predominância de indivíduos homens durante a avaliação, podendo ser justificado pela constatação de que o Transtorno do Espectro Autista é mais comum em homens do que em mulheres [Loomes et al. 2017]. Tanto [Kandalaft et al. 2012] quanto [Strickland et al. 2013] limitaram o estudo para indivíduos com Síndrome de Asperger, contribuindo para a inclusão dos mesmos no ambiente laboral, visto que sujeitos com esse diagnóstico possuem maior risco de desemprego [Taylor and Mailick 2011].

\subsubsection{Quanto a tecnologia utilizada}

Buscando ser a mais imersiva possível, a solução de [Burke et al. 2017] utilizou uma televisão de 60-70 polegadas e um computador servidor para rodar o software. Também foi utilizado o sistema de câmera do XBOX GEN 1 KINECT para rastrear expressões faciais.

Diferentemente, [Ward and Esposito 2018] apenas utilizaram o auxílio de uma televisão para familiarizar os voluntários no ambiente de intervenção através de módulos de inicialização em uma sessão em grupo. Após isso, individualmente acessaram o ambiente através de Chromebooks equipados com fones de ouvidos.

No ambiente utilizado em [Kandalaft et al. 2012], um terapeuta podia comunicarse com o voluntário utilizando um software de manipulação de voz para que a mesma combinasse com o seu avatar. Desenvolvido a partir do software Second Life, o ambiente virtual podia ser acessado através de um computador pelo voluntário. O mesmo podia controlar seu avatar através de um teclado e mouse.

Em [Strickland et al. 2013], os participantes utilizaram um computador e fones de ouvido para praticar suas habilidades e também contavam com o auxílio a distância de um terapeuta treinado, que fornecia feedbacks sobre seu desempenho em tempo real. A intervenção não imersiva de [Smith et al. 2017] também utilizou um computador simples.

Diferenciando-se dos anteriores, o estudo de [Schmidt et al. 2019] utilizou três diferentes abordagens para avaliar a usabilidade do ambiente: dois voluntários acessaram o aplicativo sem a utilização de head-mounted displays (HMD), enquanto que outros dois dispuseram do HMD de baixo custo Google Cardboard. Por fim, três dos participantes utilizaram o Google Daydream HMD juntamente com um controle remoto. 
VIII Congresso Brasileiro de Informática na Educação (CBIE 2019)

Anais do XXX Simpósio Brasileiro de Informática na Educação (SBIE 2019)

\subsubsection{Quanto as melhorias e e limitações encontradas}

[Kandalaft et al. 2012] realizaram o primeiro estudo que investigou o uso de RV para aprimorar habilidades sociais e cognitivas de jovens adultos com TEA, determinando os pontos iniciais nessa área de pesquisa. Para esses autores, as evidências preliminares demonstraram a viabilidade de intervenções utilizando RV para essa população, porém a aplicação de um questionário com respostas de 'sim' ou 'não' possivelmente desorientou os resultados.

Do mesmo modo, as limitações encontradas em [Burke et al. 2017] também referem-se ao instrumento utilizado para avaliação. Os dados obtidos indicaram que os participantes desenvolveram interesse em autopromoção e autoavaliação, além de habilidades para responder questões situacionais e comportamentais. Porém, foi aplicado um novo instrumento que não possuía testes para conferir o grau de confiabilidade ao longo do tempo e os examinadores estavam cientes do número de vezes que os participantes já haviam participado das sessões.

Em [Ward and Esposito 2018] foram assumidas limitações referentes ao pequeno número da amostra, que delimitou as generalizações dos resultados positivos obtidos. O tamanho da amostra também foi um problema encontrado em [Kandalaft et al. 2012, Smith et al. 2017, Schmidt et al. 2019]. Para [Strickland et al. 2013], a limitação da amostra foi ter sido composta apenas por homens, os quais se voluntariaram, podendo não representar todos os indivíduos do espectro.

Apesar do número de participantes no estudo de [Smith et al. 2017] ser consideravelmente maior que das outras pesquisas, o problema da amostra foi a composição a partir de pequenos grupos com diferentes diagnósticos e a realização do processo em laboratório, não determinando o número de sessões ideal de treinamento e dificultando a generalização dos resultados para a comunidade.

Por fim, [Schmidt et al. 2019] trouxeram resultados positivos para o design da experiência do usuário em ambientes em RV imersivos, tanto na visão de usuários quanto de especialistas em TEA, demonstrando ser uma aplicação viável, relevante e fácil de usar. Porém foram encontradas limitações na otimização da aprendizagem relacionadas ao equilíbrio de usabilidade e imersão, além de questões sensoriais para os indivíduos com TEA.

\section{Conclusão}

Apesar de estudos apontarem a Realidade Virtual como uma ferramenta útil para indivíduos com Transtorno do Espectro Autista [Parsons and Mitchell 2002, Kandalaft et al. 2012], a pesquisa com foco em jovens adultos pertencentes ao espectro é uma área ainda pouco abordada. Essa faixa demográfica da população autista torna-se desamparada de oportunidades, devido à falta de iniciativas para sua inserção nos âmbitos da sociedade [Shattuck et al. 2012]. Para criar um ambiente efetivo de inclusão é importante analisar os métodos já existentes.

Os maiores problemas constatados nos estudos selecionados relacionaram-se as amostras: o tamanho e a predominância de participantes do sexo masculino, apesar de o TEA ser mais comum nessa população, dificultaram a generalização dos resultados. 
VIII Congresso Brasileiro de Informática na Educação (CBIE 2019)

Anais do XXX Simpósio Brasileiro de Informática na Educação (SBIE 2019)

Outro fator que afeta a generalização é o método de condução dos estudos, visto que apenas [Smith et al. 2017] avaliaram seus efeitos reais após seis meses da intervenção, os resultados podem não ser representativos das configurações do mundo real.

Porém, ao proporcionar um ambiente seguro e controlado para o treinamento e aprimoramento de habilidades, além de aumentar a autoconfiança em diversas situações, a Realidade Virtual possui grande potencial de estudo. Desta forma, o objetivo deste artigo, de apresentar uma revisão sistemática sobre o tema e fornecer uma visão geral sobre o que já foi feito, de forma a contribuir na consolidação dessa importante abordagem para educação e apoio a essa população.

\section{Referências}

Aresti, N. and Zapirain, B. (2014). Technologies as support tools for persons with autistic spectrum disorder: A systematic review. International journal of environmental research and public health, 11:7767-7802.

Baio, J. (2014). Prevalence of autism spectrum disorder among children aged 8 yearsautism and developmental disabilities monitoring network, 11 sites, united states. Cent. Dis. Control Prev., 63:1-24.

Bontempo, T. (2009). Sensory processing patterns in high-ability adults with autism spectrum disorders in the workplace.

Burke, S., Bresnahan, T., Li, T., Epnere, K., Rizzo, A., Partin, M., M. Ahlness, R., and Trimmer, M. (2017). Using virtual interactive training agents (vita) with adults with autism and other developmental disabilities. Journal of Autism and Developmental Disorders, 48.

Constantino, J., A Davis, S., D Todd, R., K Schindler, M., M Gross, M., L Brophy, S., M Metzger, L., S Shoushtari, C., Splinter, R., and Reich, W. (2003). Validation of a brief quantitative measure of autistic traits: Comparison of the social responsiveness scale with the autism diagnostic interview-revised. Journal of autism and developmental disorders, 33:427-33.

Didehbani, N., Allen, T., Kandalaft, M., Krawczyk, D., and Chapman, S. (2016). Virtual reality social cognition training for children with high functioning autism. Computers in Human Behavior, 62:703-711.

Giannini, D. (2018). Aumenta número de crianças com autismo, segundo pesquisa. noticias.r7.com.

Hendricks, D. (2010). Employment and adults with autism spectrum disorders: Challenges and strategies for success. Journal of Vocational Rehabilitation, 32:125-134.

Kandalaft, M., Didehbani, N., Krawczyk, D., Allen, T., and Chapman, S. (2012). Virtual reality social cognition training for young adults with high-functioning autism. Journal of autism and developmental disorders, 43.

Leston, J. (1996). Virtual reality: The it perspective. The Computer Bulletin, 38.

Loomes, R., Hull, L., and Mandy, W. (2017). What is the male-to-female ratio in autism spectrum disorder? a systematic review and meta-analysis. Journal of the American Academy of Child Adolescent Psychiatry, 56. 
VIII Congresso Brasileiro de Informática na Educação (CBIE 2019)

Anais do XXX Simpósio Brasileiro de Informática na Educação (SBIE 2019)

Miller, H. and Bugnariu, N. (2016). Level of immersion in virtual environments impacts the ability to assess and teach social skills in autism spectrum disorder. Cyberpsychology, behavior and social networking, 19.

Morie, J. (1994). Inspiring the future: merging mass communication, art, entertainment and virtual environments. ACM SIGGRAPH Computer Graphics, 28:135-138.

Parsons, S. and Mitchell, P. (2002). The potential of virtual reality in social skills training for people with autistic spectrum disorders. Journal of intellectual disability research : JIDR, 46:430-43.

Robertson, G., Card, S., and Mackinlay, J. (1993). Three views of virtual reality: nonimmersive virtual reality. Computer, 26:81.

Roever, L. (2018). Pico: Model for clinical questions. Evidence-Based Medicine.

Schlosser, R. W. (2006). The role of systematic reviews in evidence-based practice, research and development. Focus.

Schmidt, M., Schmidt, C., Glaser, N., Beck, D., Lim, M., and Palmer, H. (2019). Evaluation of a spherical video-based virtual reality intervention designed to teach adaptive skills for adults with autism: a preliminary report. Interactive Learning Environments.

Shattuck, P., Narendorf, S., Cooper, B., Sterzing, P., Wagner, M., and Taylor, J. (2012). Postsecondary education and employment among youth with an autism spectrum disorder. Pediatrics, 129:1042-9.

Smith, M., Smith, J., F Fleming, M., Jordan, N., Brown, H., Humm, L., Olsen, D., and Bell, M. (2017). Mechanism of action for obtaining job offers with virtual reality job interview training. Psychiatric services (Washington, D.C.), 68:appips201600217.

Strickland, D. C., Coles, C., and Southern, L. B. (2013). Jobtips: A transition to employment program for individuals with autism spectrum disorders. Journal of autism and developmental disorders, 43.

Taylor, J. and Mailick, M. (2011). Employment and post-secondary educational activities for young adults with autism spectrum disorders during the transition to adulthood. Journal of autism and developmental disorders, 41:566-74.

Tzanavari, A., Charalambous-Darden, N., Herakleous, K., and Poullis, C. (2015). Effectiveness of an immersive virtual environment (cave) for teaching pedestrian crossing to children with pdd-nos.

Ward, D. M. and Esposito, K. (2018). Virtual reality in transition program for adults with autism: Self-efficacy, confidence, and interview skills. Contemporary School Psychology.

Wing, L. and Gould, J. (1979). Severe impairments of social-interaction and associated abnormalities in children-epidemiology and classification. Journal of autism and developmental disorders, 9:11-29. 